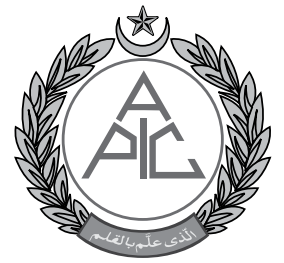

Consultant, Asian Institute of Medical Sciences,

Faridabad, Haryana, (India)

Correspondence:

Dr Jyoti Gupta

A1-801, Summer Palms, Sector

86, Neharpar, Faridabad,

Haryana-121002, (India)

Cell: +919650099128;

E-mail: jgupta79@gmail.com

Received: 15 Sep 2018

Reviewed: 18 Sep 2018

Corrected: 28 Sep, 18 Oct 2018

Accepted: 5 Nov 2018

\title{
Central venous pressure monitoring
}

\author{
Jyoti Gupta, DA, DNB
}

\begin{abstract}
The circulatory dynamics of patients are maintained by a triad of vascular capacitance, effective circulating volume and cardiac pump function. These components interact to provide blood pressure, cardiac output and tissue perfusion. Central venous pressure (CVP) is one of the parameters which is known to be useful in evaluating the interaction of these modalities. It will also help to determine volume status of the patient and need to fill in, as well as the efficacy of the volume therapy. Sometimes central line will be inserted to infuse vasoactive infusions or blood nd blood products. Its an invasive procedure and has its complications. The intensivists need to be fully conversant with the standard guidelines for this procedure for patient safety.
\end{abstract}

Key words: Central venous pressure; Monitoring; Infusions; Complications

Citation: Gupta J. Central venous pressure monitoring. Anaesth Pain \& Intensive Care 2018;22 Suppl 1:S137-S141

\section{INTRODUCTION}

The central venous pressure (CVP) measures the filling pressure of the right ventricle (RV). It gives an idea about patient's intravascular volume status. It is dependent on circulating blood volume, venous tone and right ventricular function. ${ }^{1}$

Aubaniac $^{2}$ in 1952 first advised the injection of fluids through the subclavian vessels. Later KeeriSzanto $^{3}$ in 1956 recommended the subclavian vein for intravenous injection. Wilson, ${ }^{4}$ first popularised this method in the United States in 1962.

The circulatory dynamics of a patient are maintained by the triad of vascular capacitance, effective circulating volume and cardiac pump function. These components interact to provide blood pressure, cardiac output and tissue perfusion. CVP is one of the parameters useful in evaluating the interaction of these modalities. CVP will be helpful in determining volume needs only if the assumption is valid that both ventricles are functioning with equal efficiency. Otherwise, CVP is no longer an accurate reflection of needed volume. ${ }^{5}$ Central venous catheters (CVC) are passed usually in the right subclavian or right internal jugular veins, to be used for pressure measurement and drug infusions etc.

\section{INDICATIONSFOR CVCPLACEMENT}

There have been numerous indications for a CVC placement, and it may be opted for;

1. Major operative procedures involving large fluid shifts and/ or blood loss.

2. Intravascular volume assessment when urine output is not reliable or unavailable (e.g. renal failure)

3. Major trauma

4. Surgical procedures with a high risk of air embolism such as sitting craniotomies. In addition to monitoring, the CVP catheter may also be used to aspirate intracardiac air.

5. Frequent venous blood sampling

6. Venous access for vaso-active or irritating drugs

7. Prolonged drug administration

8. Inadequate peripheral IV access

9. Rapid infusion of IV fluids

10. Special uses: 
a. Insertion of PA catheters

b. Insertion of transvenous pacing wires

c. Hemodialysis / plasmapheresis.

\section{WAVEFORMS IN CVP'}

The normal CVP waveform consists of three upward deflections (a, c and $\mathrm{v}$ waves) and two downward deflections ( $x$ and $y$ descent). These waves are produced as follows:

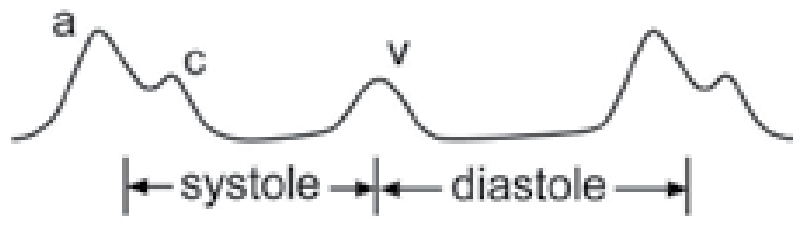

Figure 1: CVP waveform

Table 1: Abnormal CVP waveforms ${ }^{6}$

\begin{tabular}{l|l}
\multicolumn{1}{c|}{ Waveform } & \multicolumn{1}{c}{ Cardiac Abnormality } \\
\hline Absent a wave & $\begin{array}{l}\text { Atrial fibrillation } \\
\text { Sinus tachycardia }\end{array}$ \\
\hline Flutter waves & Atrial flutter \\
\hline Prominent a waves & First degree AV heart block \\
\hline Large a wave & $\begin{array}{l}\text { Tricuspid stenosis } \\
\text { Pulmonary hypertension } \\
\text { Pulmonary stenosis } \\
\text { Right atrial myxoma }\end{array}$ \\
\hline Cannon a waves & $\begin{array}{l}\text { AV dissociation } \\
\text { Ventricular tachycardia }\end{array}$ \\
\hline Absent $x$ descent & Tricuspid regurgitation \\
\hline Large cv waves & Tricuspid regurgitation \\
& Constrictive pericarditis \\
\hline Slow y descent & Tricuspid stenosis \\
& Right atrial myxoma \\
\hline Rapid y descent & Tricuspid regurgitation \\
& Atrial septal defect \\
Constrictive pericarditis
\end{tabular}

Box 1: Contraindications of CVP1

Absolute:

- $\quad$ SVC syndrome

- Infection at the site of insertion

Relative:

- Coagulopathies

- $\quad$ Newly inserted pacemaker wires

- $\quad$ Presence of carotid disease

- Recent cannulation of the internal jugular vein

- Contralateral diaphragmatic dysfunction

- Thyromegaly or prior neck surgery
1. The "a" wave is produced by right atrial contraction and occurs just after the $P$ wave on the ECG.

2. The "c" wave occurs due to isovolumic ventricular contraction forcing the tricuspid valve to bulge upward into the right atrium(RA).

3. The pressure within the RA then decreases as the tricuspid valve is pulled away from the atrium during right ventricular ejection, forming the $\mathrm{x}$ descent.

4. The RA continues to fill during the late ventricular systole forming the " $v$ " wave.

5. The ' $y$ ' descent occurs when the tricuspid valve opens and blood from the RA empties rapidly into the RV during early diastole.

\section{Technique of insertion}

There are numerous sites and techniques for central venous catheterization. Cannulation of internal jugular vein (IJV) is more popular and preferred. The reason for this popularity is:

- its landmarks,

- its short straight valve-less course to the superior vena cava and right atrium.

- its position at the patient's head provides easy access to the anaesthesiologist during the intra operative period. ${ }^{1}$

For accurate measurement of CVP/RAP and for the aspiration of air in venous air embolism, the catheter tip should be positioned ideally at the SVC-RA junction ${ }^{7}, \mathrm{SVC}^{8}$ or high up in the RA away from the tricuspid valve. ${ }^{9}$ Cannulation of the IJV is relatively safe and convenient. Various approaches exist for its cannulation.

\section{Equipment}

- Sterile mask, gloves, and gown

- Standard monitors, such as pulse oximeter, blood pressure cuff, and ECG

- When possible, peripheral IV with infusion

- Sterile prep solution (e.g., chlorhexidine)

- Sterile drapes

- 5-mL sterile syringe with 24 gauge needle for local anaesthetic infiltration

- Local anaesthetic (usually $2 \%$ lidocaine)

- 22-gauge, 1.5-inch needle: finder needle

- Central venous catheter kit containing needle, guide wire, dilator and double or triple lumen 
catheter.

- 3.0 suture on cutting needle

\section{Procedure}

Central venous catheters can be placed by two techniques:

- Landmark guided insertion

- Ultrasound-guided insertion

\section{Landmark guided technique (Right IJV)}

1. Explain the procedure to the patient and/or relatives and take written and informed consent

2. Attach basic monitors ECG, $\mathrm{SpO}_{2}$ and NIBP.

3. Remove pillow and rotate head to the left. If the patient is conscious, ask the patient to do so on his own. Note the position of sternocleidomastoid muscle and the triangle formed by its sternal and clavicular heads. If not easily appreciable, use the bony landmarks of medial end of clavicle and mastoid process.

4. Place the patient in Trendelenberg position.

5. Perform the procedure under all aseptic and antiseptic precautions.

6. Recheck landmarks. Perform local infiltration with $2 \%$ lidocaine as the patient is conscious.

7. Use a 22 Gauge 1.5 inch "finder" needle on a syringe to locate the vein.

8. Once the vein has been located, either leave the finder needle in place or remove the needle but remember the direction of vein.

9. Now, insert the needle available in the cvp kit on the syringe to locate the vein.

10. When flashback is seen, remove the syringe and pass the guidewire through the needle. Look for ectopics on ECG and avoid too deep vein (right). ${ }^{10}$ penetration. Keep a watch on vital signs.

11. Remove the needle keeping guidewire in place.

12. Pass the dilator over the guidewire. Avoid any undue pressure as it can bend the guidewire. A nick with scalpel can be made at the point of entry if it is difficult to advance the dilator smoothly.

13. Now remove the dilator and pass the pre-flushed CVP catheter over the guidewire.

14. Keep withdrawing the guidewire while inserting the CVP catheter to avoid accidental embolization. Finally, remove the guidewire completely and leave the catheter in place.

15. Secure the catheter with skin sutures.

16. Aspirate blood through all the lumens and flush them with heparinized saline. If blood is as not aspirated freely withdraw the catheter until free flow of blood occurs.

17. Place sterile dressing.
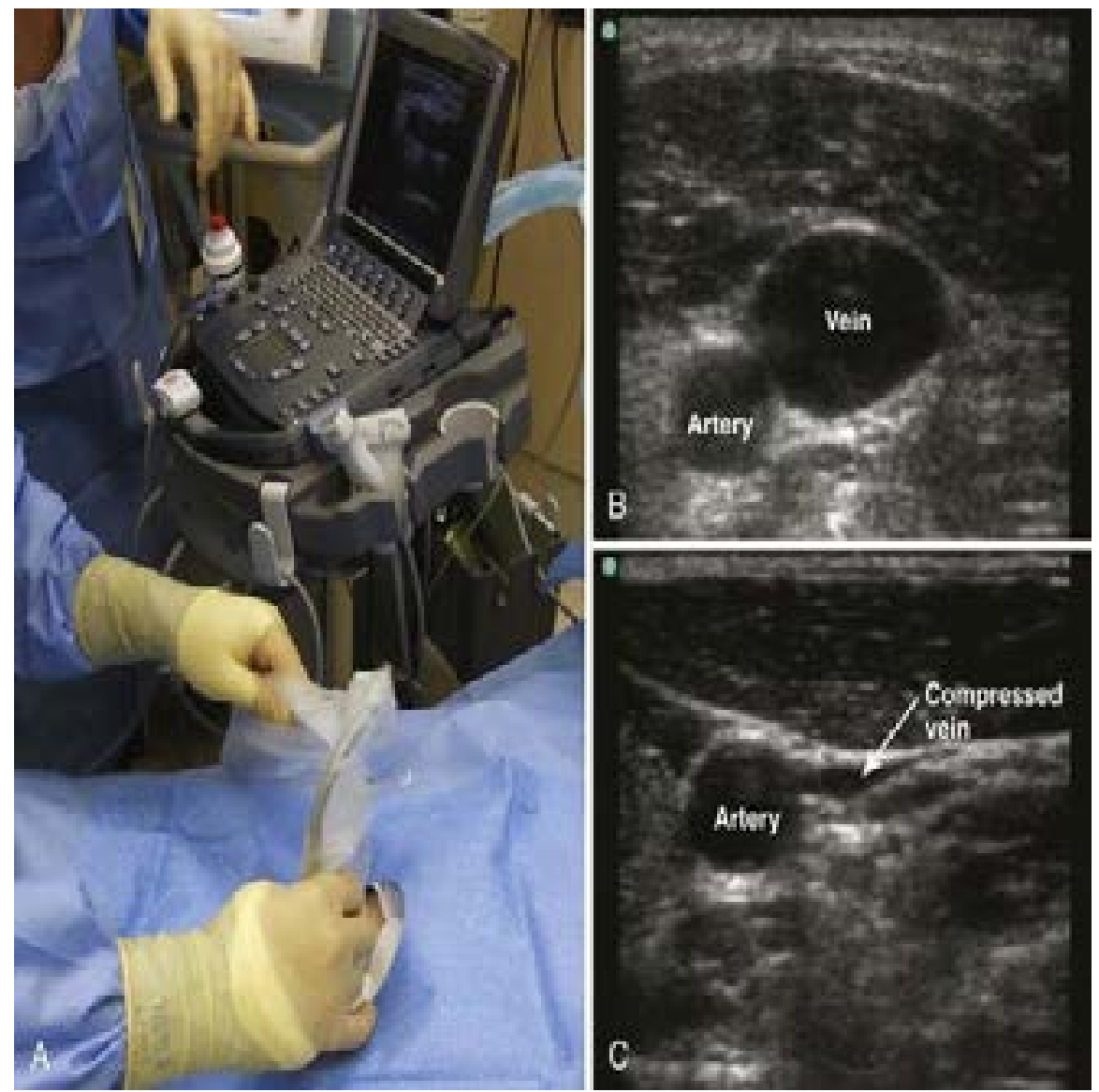

Figure 2: Ultrasound survey. Perform an ultrasound survey to identify the anatomy before beginning the procedure. $B$, Cross-sectional view of the artery (left) and noncompressed vein (right). $\mathrm{C}$, Cross-sectional view of the artery (left) and compressed 

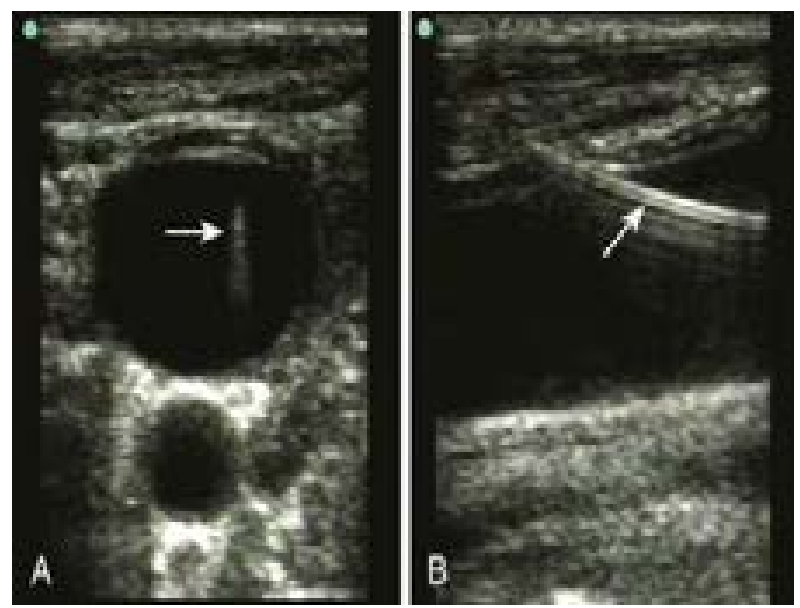

Figure 3: Cross-sectional (A) and longitudinal (B) ultrasound images demonstrating a guidewire (arrows) in the lumen of the targeted vein. ${ }^{10}$

18. Order a chest x-ray to confirm the position of catheter and rule out pneumothorax.

\section{Ultrasound guided technique}

Use of ultrasound for vascular cannulations has brought about tremendous amount of safety and success. Ultrasound-guided CVP insertion is considered as universally safe practice as it gives a real-time differentiation between IJV and carotid artery. Anatomical variations can be easily determined under USG guidance. In addition, USG guidance avoids unnecessary pricks and discomfort to the patient. Ultrasound facilitates the real time visualisation of needle, guidewire and catheter during the central venous catheterization.

\section{Pearls}

- Cannot cannulate the vein: try another site. Use ultrasound, if available

- Arterial puncture: this is not always obvious,

\section{Box 2: Complications of CVC}

\section{Complications of central venous cannulation}

- $\quad$ Arterial puncture with Hematoma

- Arteriovenous fistula

- Hemothorax

- Chylothorax

- Pneumothorax

- Nerve injury: brachial plexus or stellate ganglion

- Air embolism

- Catheter or wire shearing

\section{Complications of catheter presence}

- Thrombosis

- Thromboembolism

- Infection: sepsis, endocarditis

- Arrhythmias

- Hydrothorax especially in shocked, hypoxic patients, when the blood can be dark and flow sluggishly. If so, remove the needle and press firmly for 5 minutes with a gauze pad.

- Vein cannulated but guidewire fails to advance: sometimes veins are small, tortuous, or occluded (e.g., by thrombus from previous cannulation attempts). Do not force the wire; try another site. Use ultrasound, if available.

- Line in wrong place: occasionally, lines double back on themselves or subclavian lines go up into the jugular. If this occurs, either remove the line and start again or, if fluoroscopic guidance is available, try to reinsert the wire, remove the line, and reposition the wire.

- Beware of using too much local anaesthetic on multiple line insertions; the maximum dose should not really exceed 3-5 mg/kg (e.g., 10-15 $\mathrm{ml}$ of $2 \%$ lidocaine for a $70-\mathrm{kg}$ patient).

- Central lines do not cure patients. They take time for insertion and often require the patient to lie flat with drapes on the head for some time. Make sure that you are treating the patient's condition as well as inserting a central line.

\section{Nursing Care}

- Flush lumens on catheter with saline.

- Obtain chest radiograph to confirm position of catheter and exclude pneumothorax.

- Use sterile technique when injecting drugs or connecting tubing to lumens of catheter.

- Routinely replace sterile dressings, cleansing the site with chlorhexidine before applying a new dressing.

- Examine the insertion site daily for signs of infection.

- While the catheter is in place, leave sterile caps in place at all times and cleanse ports with alcohol before connecting anything to them.

- When preparing to remove the catheter, place the patient in Trendelenburg's position. Ask the patient to exhale as the catheter is removed, to prevent air embolism, and apply pressure over the site for 1 to 2 minutes for hemostasis.

\section{Complications of $\mathrm{CVC}^{1}$}

Numerous complications have been described; some of these are associated with cannulation process as such, while others may be a result of the presence of the catheter in the veins (Box 2). The frequency of cannulation related complications has been sharply 


\section{central venous pressure monitoring}

decreased after the wide use of ultrasonographic guidance to locate the veins and visualize the guidewire and the catheter moving inside in real time.

Universal checklist for CVC insertion and pressure monitoring are available on The American Society of Anesthesiologists website, and can be downloaded. ${ }^{11}$ It can be used to minimise the complications.

Conflict of interest: Nil declared by the author

\section{REFERENCES}

1. Muralidhar. K. Central venous pressure and pulmonary capillary wedge pressure monitoring. Indian J. Anaesth. 2002;46(4):298-303.

2. Aubaniac, R. L'injection intraveineuse sousclaviculaire: Advantages et technique. La Presse Med. 60:1456, 1952.

3. Keeri-Szanto, M. The Subclavian Vein, A Constant and Convenient Intravenous Injection Site. Arch Surg. 1956;72:179.

4. Wilson, J. N. et al. Central Venous Pressure in Optimal Blood Volume Maintenance. Arch. Surg. 1962;85:563.

5. James PM, Myers RT.: Central
Venous Pressure Monitoring: Misinterpretation, Abuses, Indications and a New Technic. Ann. Surg. 1972;175(5):693-701.

6. Cook DJ, Simel DL. Does this patient have abnormal central venous pressure? JAMA,1996;275:630-634.

7. Stanley TEIII, Reeves JG. Cardiovascular monitoring. In Miller RD, editor. Anesthesia, New-York: Churchill Livingstone;1990: p 103199.

8. Peres EPW. Positioning central venous catheter. A prospective study. Anaesth Intens Care. 1990;19:536-9.

9. Nichols PKT, Major E. Central venous cannulation. Cur Anaesth Crit Care.
1989;1:54-60.

10. McNeil CR, Rezaie SR, Adams BD. iKnowledge. 2015. Available on https://clinicalgate.com/centralvenous-catheterization-and-centralvenous-pressure-monitoring/ (Accessed on 18 October 2018)

11. Practice Guidelines for Central Venous Access: A Report by the American Society of Anesthesiologists Task Force on Central Venous Access. The American Society of Anesthesiologists, Inc. Lippincott Williams \& Wilkins. Anesthesiology 2012;116:539-73. 